\title{
AMoPCE no Ensino Superior de Programação: uma Perspectiva Docente
}

\author{
Alexandre Grotta ${ }^{1,2}$, Edmir Parada Vasques Prado ${ }^{2}$ \\ ${ }^{1}$ Instituto Federal de São Paulo (IFSP) \\ Programa de Tecnologia em Análise e Desenvolvimento de Sistemas \\ ${ }^{2}$ Universidade de São Paulo (USP) \\ Programa de Pós-graduação em Sistemas de Informação \\ grottalifsp.edu.br, eprado@usp.br
}

\begin{abstract}
Computer programming is a fundamental topic in System Analysis and related higher education courses. Given this context, the agile projectbased leaning $(A P j B L)$ is a relevant teaching method. Thus, the general objective of this research is to report the application of an APjBL method, the Agile Model for Projects in Computing Education (AMoPCE) in the Brazilian context. As a result of this research, we present the adapted version of $A M o P C E$ and its feasibility to be applied.
\end{abstract}

Resumo. A programação de computadores é um tópico fundamental em cursos superiores de Sistemas de Informação e áreas afins. Neste contexto, a aprendizagem baseada em projeto ágil (APjBL) apresenta-se como relevante alternativa de ensino. Esta pesquisa adaptou e aplicou um método APjBL, o Agile Model for Projects in Computing Education (AMoPCE), em um curso de Análise e Desenvolvimento de Sistemas. Como resultados, são apresentados a versão adaptada do AMoPCE e a facilidade percebida de sua aplicação.

\section{Introdução}

O ensino de programação de computadores é essencial em cursos superiores de Ciência da Computação, Análise e Desenvolvimento de Sistemas (ADS) e afins. Neste contexto, faz-se necessário considerar os métodos ágeis de gestão de projeto, pois estes possuem sua origem atrelada ao próprio desenvolvimento de softwares e têm sido amplamente utilizados em projetos educacionais, governamentais e da indústria de software [Petri et al. 2018; Romeike and Göttel 2012].

Assim, a aprendizagem baseada em projeto ágil (APjBL) pode contribuir com benefícios aos alunos quando comparada à aprendizagem baseada em métodos de ensino tradicionais de programação [Kastl et al. 2016]. No entanto, há relatos expectativas diferentes expectativas dos docentes bem relatos sobre a falta de padronização e dificuldades do APjBL no contexto do ensino superior internacional e também brasileiro [Grotta and Prado 2019]. Diante destes desafios, esta pesquisa relata a adaptação ao contexto brasileiro de um método APjBL, o Agile Model for Projects in Computing Education (AMoPCE), e descreve as percepções docentes com relação à facilidade para aplicação de cada um dos sete princípios que compõem o AMoPCE. 


\section{Descrição da Prática}

AMoPCE foi originalmente desenvolvido com 14 diferentes princípios e representado graficamente de duas maneiras diferentes [Romeike and Göttel 2012]. Com base no continuum educacional descrito por Biggs e Tang (2011), adaptou-se e simplificou-se o AMoPCE em sete princípios:

(1) planejamento colaborativo: Um miniprojeto é aquele projeto minimalista que é trabalhado em classe durante a aplicação do AMoPCE. Este miniprojeto é planejado por meio deste princípio.

(2) iteração: Esta é a fase na qual os participantes interagem para criação do objetivo previamente planejado: o miniprojeto. Cada iteração é composta pelas atividades de design, codificação e teste de software. É facultado aos docentes referir-se à iteração como sprint, caso possuam maior familiaridade com esta expressão.

(3) reunião em pé: reunião breve e objetiva, com limite entre cinco e quinze minutos, cujo objetivo é o planejamento das atividades do dia.

(4) programação em pares: Na programação em pares, um dos alunos assume o controle do teclado enquanto o outro contribui com direcionamentos e ideias sobre os exercícios em questão. Em determinado período, as duplas revezam-se no controle.

(5) opção pelo mais simples: Este princípio recomenda a utilização de abordagem minimalista sempre que esta atender às necessidades. Ele pode levar a diversas interpretações, tais como: utilizar códigos-fonte e métodos mais simples; fazer primeiro as atividades menores; entre outras.

(6) jogo do planejamento: os participantes contribuem com estimativas de algo relevante para o projeto. É comum a associação do jogo do planejamento com estimativas de tempo, ou seja, estimar a duração das tarefas do projeto por exemplo.

(7) apresentações: É a apresentação final do miniprojeto, na forma de protótipo ou de produto operante. Mais especificamente, de modo incremental, cada apresentação vai mostrando a evolução de protótipo em relação ao produto final, no contexto da disciplina. Cada apresentação resulta reflexões que ajudam no próximo planejamento colaborativo.

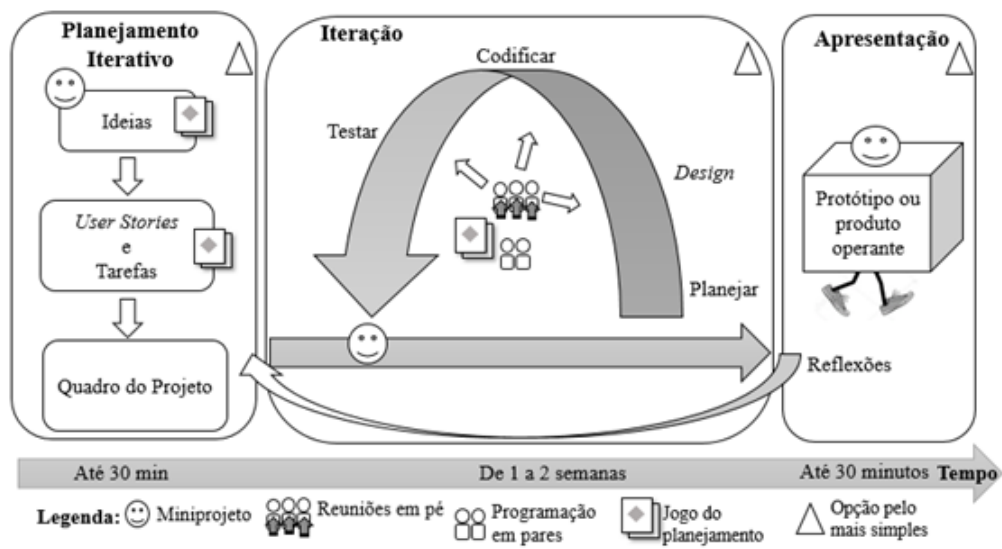

Figure 1. AMoPCE adaptado ao contexto desta pesquisa 


\section{Resultados}

A aplicação do AMoPCE foi realizada no primeiro semestre de 2018 nas disciplinas de programação de um curso superior em tecnologia de ADS no interior do Estado de São Paulo. Ao término das aulas, foram realizadas as entrevistas com os professores. As disciplinas eram compostas por quatro aulas semanais, ofertadas em um mesmo dia, perfazendo 20 semanas letivas. Participaram desta pesquisa cinco professores e 151 alunos, distribuídos em 12 turmas. Estes foram divididos em grupos de controle e experimento, com a seguinte configuração: Foram três grupos de experimento, sendo uma disciplina grupo, em um total 41 alunos; três grupos de controle, compostos por duas ou mais disciplinas, em um total 110 alunos. A tabela 1 sumariza a percepção da facilidade da aplicação dos princípios AMoPCE na perspectiva dos docentes participantes:

Table 1. Facilidade percebida para aplicação do AMoPCE

\begin{tabular}{|l|c|c|c|}
\hline $\begin{array}{l}\text { Disciplina de Experimento } \\
\text { Princípio }\end{array}$ & $\begin{array}{c}\text { Linguagem de } \\
\text { Programação }\end{array}$ & Estrutura de Dados & $\begin{array}{c}\text { Dispositivos } \\
\text { Móveis }\end{array}$ \\
\hline Planejamento colaborativo & Intermediário & Fácil & Fácil \\
\hline Iteração & Intermediário & Difícil & nácil \\
\hline Apresentações & Fácil & Intermediário & Fácil \\
\hline Reuniões em pé & Fácil & não utilizado & não utilizado \\
\hline Programação em pares & Difícil & Intermediário & Difícil \\
\hline Opção pelo mais simples & Intermediário & não utilizado & não utilizado \\
\hline Jogo do Planejamento & Fácil & & \\
\hline
\end{tabular}

\section{Recomendações}

Com base na percepção dos docentes participantes e do pesquisador, visando resultados mais assertivos em futuras aplicações do AMoPCE, recomenda-se primeiramente um planejamento docente antecipado. Neste planejamento, escolhe-se ao menos dois princípios dos sete disponíveis. Após a implementação bem-sucedida destes dois, devese escolher outros dois princípios para serem implementados ao longo do semestre, totalizando assim um mínimo de quatro princípios aplicados ao longo de no mínimo duas sprints. A utilização de um único princípio, por exemplo, dado o continuum educacional, o método de ensino aproximar-se-á daquele predominantemente utilizado em sala, que no caso não será $\mathrm{APjBL}$.

AMoPCE foi bem recebido nas disciplinas de qualquer período do curso. Não foram encontradas limitações relacionadas a alunos calouros, intermediários ou veteranos. No entanto, cabe destacar que as turmas de início do curso mostraram-se mais propensas a aceitar o AMoPCE. Os primeiros semestres foram percebidos como os melhores para ser criar uma cultura de aprendizagem ativa, com foco no APjBL. Por outro lado, em disciplinas intermediárias, as turmas já possuíam uma cultura mais propensa a esperar pelo conhecimento - esperando-se uma aprendizagem narrada como pronta e acabada. Ainda assim, a percepção foi de que o AMoPCE beneficiou os alunos no sentido que contribuiu para ser tornarem mais ativos e atuantes em sala de aula, e espera-se, por consequência, serem mais atuantes na sociedade e na vida profissional. 
Recomenda-se também que o AMoPCE seja aplicado a disciplinas de conteúdo predominantemente prático, dada a maior dificuldade, constatada nesta pesquisa, da aplicação do AMoPCE em disciplinas predominantemente conceituais. Recomenda-se, por conseguinte, que futuras pesquisas abordem esta lacuna, a dificuldade de aplicação AMoPCE em disciplinas teóricas.

\section{Conclusão}

Esta pesquisa relatou a adaptação de um método APjBL, o AMoPCE, ao contexto do ensino superior brasileiro, mais especificamente em um curso superior de ADS para disciplinas de programação de computadores. Esta pesquisa faz parte de um projeto maior sobre a utilização da APjBL no ensino superior brasileiro e teve por foco a percepção dos docentes a respeito da facilidade de aplicação de cada um dos sete princípios do AMoPCE.

AMoPCE mostrou-se versátil pois foi aplicável desde semestres iniciais do curso até os semestres finais, bem como aplicável a diferentes níveis de complexidade das disciplinas, desde as introdutórias até as mais complexas. AMoPCE apresentou melhor desempenho em disciplinas mais práticas (procedurais) e em turmas mais participativas, que, no contexto da pesquisa, foram representadas por turmas de alunos calouros e de veteranos.

\section{Referências}

Biggs, J. B. and Tang, C. S. (2011). Effective teaching and learning for today's universities. Teaching for quality learning at university. SRHE and Open University Press Imprint. 4. ed. New York, USA: McGraw-Hill Education. p. 1-16.

Grotta, A. and Prado, E. P. V. (2019). Project Management Approaches and Methods to Computer Programming Education Via Project-Based Learning: A Systematic Literature Review. In PBL2019 IMMERSIVE VIRTUAL INTERNATIONAL CONFERENCE. . http://pbl2019.panpbl.org/wpcontent/uploads/2019/09/AlexandreGrotta-AprendizagemBaseadaemProjeto.pdf.

Kastl, P., Kiesmüller, U. and Romeike, R. (2016). Starting out with projects Experiences with agile software development in high schools. [B. E. Vahrenhold J. Barendsen E., Ed.]In: Workshop in Primary and Secondary Computing Education, 4th. Association for Computing Machinery. https://www.scopus.com/inward/record.uri?eid=2-s2.0$84994322700 \&$ doi $=10.1145 \% 2 F 2978249.2978257 \&$ partnerID=40\&md5=fcbaa46e 9 $5 \mathrm{~d} 7 \mathrm{~d} 0 \mathrm{cdbfaf} 1 \mathrm{~b} 0 \mathrm{fc} 3 \mathrm{~b} 78 \mathrm{c} 3 \mathrm{f}$.

Petri, G., Calderón, A., Von Wangenheim, C. G., Borgatto, A. F. and Ruiz, M. (2018). Benefícios dos Jogos Não-Digitais no Ensino de Computação. In $26^{\circ}$ Workshop sobre Educação em Computação (WEI 2018). . SBC. http://portaldeconteudo.sbc.org.br/index.php/wei/article/view/3481.

Romeike, R. and Göttel, T. (2012). Agile projects in high school computing education Emphasizing a learners' perspective. In: Proceedings of the 7th Workshop in Primary and Secondary Computing Education. 